Pacific Journal of Mathematics

GENERALIZED SPECTRAL OPERATORS ON LOCALLY Cun-Yuni Ma 


\title{
GENERALIZED SPECTRAL OPERATORS ON LOCALLY CONVEX SPACES
}

\author{
Fumi-Yuki Maeda
}

Introduction. The theory of spectral operators on Banach spaces has been successfully generalized to a certain class of locally convex spaces by Ionescu Tulcea [4], using the definition of spectra introduced by Waelbroeck [7]. On the other hand, Foias [3] considered a notion of generalized scalar operators on Banach space. The present work, suggested by these two works, develops a theory of very general spectral operators on locally convex space for which the space of linear continuous operators is quasi-complete.

Let $A$ be a complex algebra with unit 1 endowed with a topology which makes $A$ a separated locally convex linear space and for which the mapping $(x, y) \rightarrow x y$ is separately continuous. Given an element $x$ of $A$, the resolvent set $\rho(x)$ of $x$ is a subset of $\hat{C}=C \cup\{\infty\}$ (the one point compactification of the complex plane $C$ ) consisting of all points $\lambda$ in $\hat{C}$ such that there is a neighborhood $V_{\lambda}$ of $\lambda$ in which $\mu 1-x$ is invertible in $A$ for $\mu \in V_{\lambda}-\{\infty\}$ and the set $\left\{(\mu 1-x)^{-1} ; \mu \in V_{\lambda}-\{\infty\}\right\}$ is bounded in $A$. $s p(x)=\widehat{C}-\rho(x)$ is called the spectrum of $x$. If $\infty \notin s p(x)$, then $x$ is called regular. One may refer to [7] and [5] for more details.

Waelbroeck [7] showed that if $A$ is quasi-complete and $x$ is a regular element of $A$, then the formula

$$
f(x)=(1 / 2 \pi i) \int_{\gamma} f(\lambda)(\lambda 1-x)^{-1} d \lambda
$$

defines an element $f(x)$ of $A$ for any function $f$ holomorphic in a neighborhood of $s p(x)$, where $\gamma$ is a closed contour surrounding $s p(x)$ and contained in the domain where $f$ is holomorphic. Let $D$ be an open set containing $s p(x)$ and let $H(D)$ be the space of all holomorphic functions on $D$. Then the mapping $f \rightarrow f(x)$ is a continuous homomorphism (linear multiplicative mapping) of $H(D)$ into $A$. The function $f(\lambda) \equiv 1$ is mapped to 1 and the function $f(\lambda) \equiv \lambda$ is mapped to $x$. Conversely, if there is a continuous homomorphism $u$ of $H(D)$ into $A$ such that $u(1)=1$ and $u(\lambda)=x$, then $u(f)=f(x)$ for all $f \in H(D)$.

If $A$ is an algebra given above, we have an isomorphism $x \rightarrow L_{x}$ of $A$ into $\mathscr{L}(A)$ defined by $L_{x} y=x y$ for all $y \in A$. Here, $\mathscr{L}(A)$ is the algebra of all continuous linear operators. The isomorphism is topological, if we consider the simple convergence topology in $\mathscr{L}(A)$. Furthemore, if $L_{x}$ is invertible in $\mathscr{L}(A)$, then so is $x$ in $A$. Therefore, the spectral

\footnotetext{
Received March 11, 1962.
} 
theory on $A$ can be reduced to that on $\mathscr{L}(A)$, or more generally, to that on $\mathscr{L}(E)$, where $E$ is a separated locally convex space such that $\mathscr{L}(E)$ contains a quasi-complete full subalgebra with respect to some topology.

Thus, we shall consider in this paper a locally convex space $E$ for which $\mathscr{L}(E)$ is quasi-complete with respect to the uniform convergence topology on bounded sets in a family $\mathfrak{S}$; $\mathfrak{S}$ is a collection of bounded sets whose union is the whole space $E$. We remark that in this case, $E$ itself is quasi-complete. Ionescu Tulcea [4] defined a scalar operator $S \in \mathscr{L}(E)$ as follows: Suppose there is a family $\mathscr{F}=\left\{\mu_{x, x^{\prime}}\right\}_{x \in E, x^{\prime} \in E^{\prime}}$ of bounded complex Radon measures on $C$ and suppose there exists a continuous homomorphism $f \rightarrow U(f)$ of the algebra of all bounded complex-valued Borel measurable functions on $C$ into the algebra $\mathscr{L}(E)$ such that $U(1)=I$ and $\int f d \mu_{x, x^{\prime}}=\left\langle U(f) x, x^{\prime}\right\rangle$ for all $x \in E, x^{\prime} \in E^{\prime}$. If the support of $\mathscr{F}\left(=\right.$ the closure of the union of all supp $\left.\mu_{x, x^{\prime}}, x^{\prime} \in E^{\prime}\right)$ is compact and if $\left\langle S x, x^{\prime}\right\rangle=\int \lambda d \mu_{x, x^{\prime}}(\lambda)$ for all $x \in E, x^{\prime} \in E^{\prime}$, then we say that $S$ is scalar. If, in this case, $f$ is holomorphic in a neighborhood of $s p(S)$, then we see that $f(S)=U(f)$. Hence the mapping $f \rightarrow U(f)$ can be regarded as a linear multiplicative extension of the mapping $f \rightarrow f(S)$. Similarly, a generalized scalar operators $S$ introduced by Foias [3] is an operator in $\mathscr{L}(E)$ for which there is a continuous homomorphism $U$ of $\mathscr{C}^{\infty}(C)$ into $\mathscr{L}(E)$ such that $U(1)=I$ and $U(\lambda)=S$. Here again, the mapping $f \rightarrow U(f)$ is an extension of the mapping $f \rightarrow f(S)$. These observations motivate us to define very general scalar operators (Definition 1.2) and furthermore, general spectral operators of transformations (Definitions $3.1 \& 3.2$ ). We will see that many properties of scalar and spectral operators given in [1], [2], [3], [4], which called for more restricted conditions, remain true for our generalized ones. In particular, the decomposition theorem on spectral operators into scalar and quasi-nilpotent parts will be given in the form of Theorem 4.1 and 4.2 for the generalized spectral transformations. One should remark that only significant condition put on $E$ is that $\mathscr{L}(E)$ is quasi-complete.

Finally, it is well to mention that a linear difierential operator with constant coefficients defined on the space of rapidly decreasing functions on $R^{n}$ is a generalized scalar operator by our definition (Example 2.5).

\section{1. $\Phi$-scalar operators and $\Phi$-spectral representations.}

We consider an algebra $\Phi$ of Borel measurable complex valued functions on $C\left(=R^{2}\right)$. If $\sigma$ is a Borel set in $C$, then the set of all $f \in \Phi$ such that supp $f \subseteq \sigma$ will be denoted by $\Phi_{\sigma}$ and the set of all $f \in \Phi$ with compact support is denoted by $\Phi_{c}$.

Definition 1.1. An algebra $\Phi$ of functions is called basic if it has 
the following properties:

(i) For any compact set $\delta(\subseteq C)$ and an open set $\sigma \supseteqq \delta$, there is a function $f \in \Phi$ such that $f \equiv 1$ on a neighborhood of $\delta, f \equiv 0$ outside of $\sigma$ and $0 \leqq f \leqq 1$.

(ii) If $f \in \Phi$ has a compact support and if $\varphi$ is holomorphic in a neighborhood of supp $f$, then $f \varphi \in \Phi$.

(iii) $\Phi$ is a separated topological algebra with a topology stronger than that of uniform convergence on each compact set; $\Phi_{c}$ is dense in $\Phi$; the mapping $\varphi \rightarrow f \varphi$ is continuous from $H(\sigma)$ into $\Phi$ for any open set $\sigma$ containing supp $f, f \in \Phi_{c}$.

Examples of basic algebras

(1) $\mathscr{B}=\mathscr{B}(C)=$ the algebra of all locally bounded Borel measurable functions on $C$ with the topology of uniform convergence on each compact set.

(2) $\mathscr{B}_{c}=\mathscr{B}_{c}(C)$. The topology is the inductive limit topology of क्षं's.

(3) $\mathscr{C}^{0}=\mathscr{C}^{0}(C)=$ the set of all continuous functions with the same topology as $\mathscr{B}$.

(4) $\mathscr{C}_{c}^{0}=\mathscr{C}_{c}^{0}(C)$. The inductive limit topology of $\mathscr{C}_{\delta}^{0}$ 's is given.

(5) $\mathscr{C}^{\infty}=\mathscr{C}^{\infty}(C)=$ the set of all infinitely differentiable functions with the topology of uniform convergence of all derivatives on each compact set.

(6) $\mathscr{C}_{c}^{\infty}=\mathscr{C}_{c}^{\infty}(C)$ is the space $\mathscr{D}\left(R^{2}\right)$ of $L$. Schwartz [6].

Given a basic algebra $\Phi$ and an open set $\sigma$, let $H(\Phi ; \sigma)=$ $\{f \in \Phi ; f / \sigma \in H(\sigma)\}=\{f \in \Phi ; f$ is holomorphic in $\sigma$.$\} Then the mapping$ $f \rightarrow f / \sigma$ is a continuous homomorphism of $H(\Phi ; \sigma)$ into $H(\sigma)$ by (iii) of Definition 1.1.

Throughout this paper, let $E$ be a separated locally convex space over $C$ such that $\mathscr{L}(E)$ is quasi-complete with respect to a topology of uniform convergence on bounded sets in a family $\mathfrak{S}$. We always consider this given topology unless otherwise specified. Then, $E$ itself is quasi-complete and any set in $\mathscr{L}(E)$ which is bounded for the simple convergence topology is bounded for the given topology. The set of all regular elements, i.e., the set of all $T \in \mathscr{L}(E)$ with compact spectrum, will be denoted by $\mathscr{L}_{r}(E)$.

Given $T \in \mathscr{L}_{r}(E)$ and an open set $\sigma \supset s p(T), \varphi(T) \in \mathscr{L}(E)$ is defined for any $\varphi \in H(\sigma)$. (See the introduction.) Therefore, $f(T) \equiv(f / \sigma)(T)$ is defined as an element of $\mathscr{L}(E)$ for each $f \in H(\Phi ; \sigma)$. The mapping $f \rightarrow f(T)$ is a continuous homomorphism of $H(\Phi ; \sigma)$ into $\mathscr{L}(E)$.

Definition 1.2. $T \in \mathscr{L}_{r}(E)$ is called $\Phi$-scalar if the mapping $f \rightarrow f(T)$ of $H(\Phi ; \sigma)$ into $\mathscr{L}(E)$ can be extended to a continuous homomorphism of $\Phi$ into $\mathscr{L}(E)$ for any open set $\sigma$ containing $\operatorname{sp}(T)$. 
DeFINITION 1.3. A continuous homomorphism $U$ of $\Phi$ into $\mathscr{L}(E)$ such that the identity is in the weak closure (i.e., the closure with respect to the simple convergence topology) of the image of $U$ in $\mathscr{L}(E)$ is called a $\Phi$-spectral representation.

Proposition 1.1. If $U$ is a $\Phi$-spectral representation, then $\{U(f) x ; f \in \Phi, x \in E\}$ is dense in $E$. In fact, there is a net $\left\{f_{a}\right\}$ of functions $f_{\alpha} \in \Phi$ with compact support such that $U\left(f_{\alpha}\right) x \rightarrow x$ for all $x \in E$.

Proof. Since the identity $I$ is in the weak closure of the image of $U$, there is a net $\left\{f_{\alpha}\right\}$ of functions in $\Phi$ such that $U\left(f_{\alpha}\right) x \rightarrow x$ for all $x \in E$. Since $\Phi_{c}$ is dense in $\Phi$ and since $U$ is continuous, we can choose $f_{\alpha}$ 's having compact supports.

Proposition 1.2. If $U$ is a $\Phi$-spectral representation and if constants are in $\Phi$, then $U(1)=I$ and hence

$$
\{U(f) x ; f \in \Phi, x \in E\}=E .
$$

Proof. $U(1) U(f) x=U(f) x$ for all $f \in \Phi, x \in E$. Hence by Proposition 1.1, $U(1)=I$.

Proposition 1.3. Let $U$ be a $\Phi$-spectral representation with compact support. Let $\delta_{0}=\operatorname{supp} U$ and let $f_{0} \in \Phi$ be equal to 1 in a neighborhood of $\delta_{0}$. Then, $U\left(f_{0}\right)=I$ and $S=U\left(\lambda f_{0}\right)\left(\lambda f_{0}\right.$ is the function $\lambda f_{0}(\lambda)$. It is an element of $\Phi$ by the condition (ii) of Definition 1.1.) is $\Phi$-scalar and $s p(S)=\delta_{0}$. In fact, $U$ is an extension map of $f \rightarrow f(S)$.

Proof. For any $f \in \Phi$, supp $\left(f-f_{0} f\right)=C \delta_{0}$. Hence $U\left(f-f_{0} f\right)=0$ or $U(f)=U\left(f_{0}\right) U(f)$. Hence by Proposition 1.1 we have $U\left(f_{0}\right)=I$. Thus, if we know that $s p(S)=\delta_{0}$, then we obtain $U(f)=f(S)$ for $f \in H(\Phi ; \sigma)$ for any $\sigma \supset \operatorname{sp}(S)$ from the uniqueness of $f(S)$ and the proposition will be proved. The fact that $s p(S)=\delta_{0}$ can be shown in the way of the proof of Proposition 1, Foias [3]. We remark that if $\mu \notin \operatorname{supp} f_{0}$, then $(\mu-\lambda)^{-1} f_{0}(\lambda) \in \Phi$ by the condition (ii) of Definition 1.1 and it is holomorphic in $\mu$.

This proposition and the following one, which is immediate from the definition, show the direct relationship between $\Phi$-scalar operators and $\Phi$-spectral representations.

Proposition 1.4. If $S$ is $\Phi$-scalar, then an extension map $f \rightarrow U(f)$ of $f \rightarrow f(S)$ is a $\Phi$-spectral representation and hence $\operatorname{sp}(S)=\operatorname{supp} U$ (by the previous proposition). 
REMARK 1. Let $\Phi_{1}$ and $\Phi_{2}$ be two basic algebras. If $\Phi_{1} \subseteq \Phi_{2}$ and the topology of $\Phi_{1}$ is stronger than that of $\Phi_{2}$ induced on $\Phi_{1}$ and $\Phi_{2}$ is dense in $\Phi_{2}$, then any $\Phi_{2}$-spectral representation is $\Phi_{1}$-spectral, so that any $\Phi_{2}$-scalar operator is $\Phi_{1}$-scalar. In particular, any $\mathscr{B}$-scalar operator is $\mathscr{C}^{0}$ and $\mathscr{C}^{\infty}$-scalar and any $\mathscr{B}_{c}$-scalar operator is $\mathscr{C}_{c}^{0}$-and $\mathscr{C}_{c}^{\infty}$-scalar.

REMARK 2. It can be shown that if $E$ is reflexive, then any $\mathscr{C}^{0}$ spectral representation or $\mathscr{C}_{c}^{0}$-spectral representation is $\mathscr{B}$-spectral.

REMARK 3. If $S \in \mathscr{L}(E)$ is $\mathscr{B}$-scalar, then the family $\left\{\mu_{x, x^{\prime}}\right\}$ of complex Radon measures defined by $\mu_{x, x^{\prime}}(f)=\left\langle U(f) x, x^{\prime}\right\rangle$ for $f \in \mathscr{B}$, $x \in E$ and $x^{\prime} \in E^{\prime}$, where $U$ is an extension map of $f \rightarrow f(S)$, is a spectral family of measures corresponding to $S$ in the sense of Ionescu Tulcea [4]. Therefore, if $E$ is barreled and if we consider the topology of uniform convergence on each bounded set, the extension map $U$ must be unique by [4].

On the other hand, the following example shows that the extension may not be unique to $\mathscr{C}^{\infty}$ when $S$ is $\mathscr{C}^{\infty}$-scalar: Let $S$ be $\mathscr{C}^{\infty}$-scalar with an extension $f \rightarrow U(f)$ of $f \rightarrow f(S)$ to $\mathscr{C}^{\infty}$. Let $Q \neq 0$ be a nilpotent operator in $\mathscr{L}(E)$, say $Q^{k+1}=0$, commuting with all $U(f)$, $f \in \mathscr{C}^{\infty}$. Now, we define

$$
V(f)=U(f)+U(D f) Q+U\left(D^{2} f\right) Q^{2} / 2 !+\cdots+U\left(D^{k} f\right) Q^{k} / k !,
$$

where $D=\partial / \partial \xi_{1}+i \partial / \partial \xi_{2}\left(\lambda=\xi_{1}+i \xi_{2}\right)$. If $f$ is holomorphic in a neighborhood of $s p(S)=\operatorname{supp} U$, then $D f=0$ there, so that $V(f)=U(f)=f(S)$. It is elementary to see that $V$ is a homomorphism of $\mathscr{C}^{\infty}$ into $\mathscr{L}(E)$. Since $f \rightarrow D f$ is continuous in $\mathscr{C}^{\infty}, f \rightarrow V(f)$ is continuous. Thus, $V$ is an extension of $f \rightarrow f(S)$ to be a continuous homomorphism on $\mathscr{C}^{\infty}$ and is different from $U$.

DeFinition 1.4. Let $X$ be a set and let $\Psi$ be an algebra of complex valued functions on $X$ with a locally convex topology. Given a basic algebra $\Phi, \Psi$ is called $\Phi$-admissible if

(i) $f \circ \varphi \in \Psi$ for any $f \in \Phi$ and $\varphi \in \Psi$, and

(ii) $f \rightarrow f \circ \varphi$ is a continuous mapping of $\Phi$ into $\Psi$ for each $\varphi \in \Psi$.

We remark that (i) implies that $\Psi$ contains constant functions. The following examples of $\Phi$-admissible algebra are of special interest:

EXAMPLE 1.1. Let $X$ be a separated topological space and let $\Psi$ be the set of all bounded continuous functions on $X$ with the uniform convergence topology. Then $\Psi$ is $\mathscr{C}_{c}^{0}$-and $\mathscr{C}^{0}$-admissible.

ExAmple 1.2. Let $X$ be a locally compact space and let $\Psi$ be the topological direct sum of two algebras $\Psi_{1}$ and $C ; \Psi=\Psi_{1} \oplus C$, where 
$\Psi_{1}$ is the set of all continuous functions with compact support. The topology of $\Psi_{1}$ is the inductive limit topology of $\Psi_{1, \varepsilon}$ 's like that of $\mathscr{C}_{0}^{0}$. Then $\Psi$ is $\mathscr{C}_{c}^{0}$-admissible. In fact, if $f \in \mathscr{C}_{c}^{0}$, then $f \circ \varphi=$ $\{f \circ \varphi-f(0)\}+f(0)$ and $f \circ \varphi-f(0) \in \Psi_{1}$. Furthermore we have

$$
\|f \circ \varphi-f(0)\|_{\text {supp } \varphi} \leqq\|f\|_{\delta}+|f(0)| \leqq 2\|f\|_{\delta \cup\{0\}},
$$

where $\delta=\overline{\varphi(X)}$.

ExAmple 1.3. Let $X$ be a $\mathscr{C}^{\infty}$-manifold and $\Psi$ be the set of all $\mathscr{C}^{\infty}$-functions on $X$ whose derivatives are all bounded. If we consider the topology of uniform convergence of all derivatives in $X$, then $\Psi$ is $\mathscr{C}^{\infty}$-and $\mathscr{C}_{c}^{\infty}$-admissible.

Example 1.4. Let $X$ be a $\mathscr{C}^{\infty}$-manifold and let $\Psi_{1}$ be the set of all $\mathscr{C}^{\infty}$-functions with compact support. The topology of $\Psi_{1}$ is the inductive limit topology like that of $\mathscr{C}_{c}^{\infty}$. Then $\Psi=\Psi_{1} \oplus C$ is $\mathscr{C}_{c}^{\infty}-$ admissible.

THEOREM 1.1. Let $\Phi$ be a basic algebra and let $\Psi$ be a $\Phi$-admissible algebra of functions on $X$. If $V$ is a continuous homomorphism of $\Psi$ into $\mathscr{L}(E)$ such that $V(1)=I$, then $V(\varphi)$ is $\Phi$-scalar whenever $\varphi \in \Psi$ is bounded, $s p(V(\varphi)) \subseteq \overline{\varphi(X)}$ and, in fact, $f \rightarrow V(f \circ \varphi)$ is an extension map of $f \rightarrow f(V(\varphi))$.

Proof. It is clear that $f \rightarrow V(f \circ \varphi)$ is a homomorphism of $\Phi$ into $\mathscr{L}(E)$. Let $U(f)=V(f \circ \varphi)$. Since $f \rightarrow f \circ \varphi, f \circ \varphi \rightarrow V(f \circ \varphi)$ are both continuous, $f \rightarrow U(f)$ is continuous. If $f_{0} \equiv 1$ on a neighborhood of $\overline{\varphi(X)}$, then $f_{0} \circ \varphi \equiv 1$ and $\left(\lambda f_{0}\right) \circ \varphi \equiv \varphi$. Therefore, $U\left(f_{0}\right)=I$ and $U\left(\lambda f_{0}\right)=V(\varphi)$. Thus, $U$ is a $\Phi$-spectral representation. It is easy to see that supp $U \subseteq \overline{\varphi(X)}$. Therefore, by Proposition 1.3, $V(\varphi)=U\left(\lambda f_{0}\right)$ is $\Phi$-scalar, $s p(V(\varphi))=\operatorname{supp} U \subseteq \overline{\varphi(X)}$ and $f \rightarrow U(f)=V(f \circ \varphi)$ is an extension of $f \rightarrow f(V(\varphi))$.

CoRollaRY. If $\Phi$ is any one of the algebras given in the examples after Definition 1.1 and if $U$ is a $\Phi$-spectral representation, then $U(f)$ is $\Phi$-scalar for any bounded function $f \in \Phi$.

Proof. If $\Phi=\mathscr{B}, \mathscr{C}^{0}$ or $\mathscr{C}^{\infty}$, then $\Phi$ itself is $\Phi$-admissible and if $\Phi=\mathscr{B}_{c}, \mathscr{C}_{c}^{0}$ or $\mathscr{C}_{c}^{\infty}$, then $\Psi=\Phi \oplus C$ is $\Phi$-admissible, by Examples 1.2 and 1.4. Hence, taking $V(f+\alpha)=U(f)+\alpha I$ for the latter case, $U(f)=V(f)$ is $\Phi$-scalar by the theorem.

2. Subspaces corresponding to a $\Phi$-spectral representation. Given a $\Phi$-spectral representation $U$, we consider (see [3]) subspaces $E_{U, \sigma}$ of $E$ 
for open or closed set $\sigma$ in $C$ as follows:

Definition 2.1. If $\sigma$ is an open set in $C$, we define

$$
E_{U, \sigma}=\bigcup_{f \in \Phi_{\sigma}} U(f)(E)=\left\{U(f) x ; f \in \Phi_{\sigma}, x \in E\right\} .
$$

If $\delta$ is a compact set in $C$, then we define

$$
E_{U, \delta}=\bigcap\left\{E_{U, \sigma} ; \sigma: \text { open set such that } \sigma \supset \delta\right\} .
$$

The following properties are easy to verify;

(i) If $\sigma$ is an open set, then $E_{U, \sigma}$ is a linear subspace of $E$; if $\delta$ is a compact set, then $E_{U, \delta}$ is a closed linear subspace of $E$.

(ii) Let $\sigma_{1}$ and $\sigma_{2}$ be either open or compact. If $\sigma_{1} \cap \sigma_{2}=\phi$, then $E_{U, \sigma_{1}} \cap E_{U, \sigma_{2}}=\{0\}$; if $\sigma_{1} \subseteq \sigma_{2}$, then $E_{U, \sigma_{1}} \subseteq E_{U, \sigma_{2}}$.

(iii) $E_{U, \infty}=\bigcup\left\{E_{U, \delta} ; \delta\right.$ : compact $\}$ is dense in $E$. If supp $U=\delta_{0}$ is compact, then $E=E_{U, \delta_{0}}$.

The property (iii) follows from proposition 1.1 and 1.3.

Proposition 2.1. If $f \in \Phi$ is equal to 1 in a neighborhood of a compact set $\delta$, then $U(f) x=x$ for all $x \in E_{U, \delta}$.

Proof. Let $f \equiv 1$ on $\sigma$, an open set containing $\delta$. If $x \in E_{\sigma, \delta}$, then there is $g \in \Phi_{\sigma}$ and $y \in E$ such that $x=U(g) y$. Then, $U(f) x=$ $U(f) U(g) y=U(f g) y=U(g) y=x$.

Proposition 2.2. $x \in E_{U, \delta}$ if and only if $U(f) x=0$ for any $f$ such that supp $f \cap \delta=\phi$. ( $\delta$ is a compact set.)

Proof. Suppose $x \in E_{U, \delta}$. Then, there is $g \in \Phi_{\sigma}$ such that $x=$ $U(g) y$, where $\sigma=C \operatorname{supp} f$. Hence $U(f) x=U(f) U(g) y=U(0) y=0$. Conversely, suppose $U(f) x=0$ for any $f$ such that $\operatorname{supp} f \cap \delta=\phi$. Let $\sigma$ be an arbitrary open set (bounded) containing $\delta$ and let $f_{\sigma} \in \Phi_{\sigma}$ be equal to 1 in a neighborhood of $\delta$. Suppose first that $x \in E_{U, \infty}$. Then there is a compact set $\delta_{1}, \delta_{1} \supseteqq \bar{\sigma}$, such that $x \in E_{U, \delta_{1}}$. Let $f_{0} \in \Phi$ be equal to 1 in a neighborhood of $\delta_{1}$. Then, $U\left(f_{0}\right) x=x$ by Proposition 2.1. On the other hand, $f_{\sigma}-f_{0} \equiv 0$ on a neighborhood of $\delta$, i.e., $\operatorname{supp}\left(f_{\sigma}-f_{0}\right) \cap \delta=\phi$. Hence, by our assumption, $U\left(f_{\sigma}-f_{0}\right) x=0$ or $U\left(f_{\sigma}\right) x=U\left(f_{0}\right) x=x$. Therefore, $x \in E_{U, \sigma}$. Since $\sigma \supset \delta$ is arbitrary, $x \in E_{U, \delta}$. Next, let $x$ be arbitrary. Take a net $\left\{f_{\alpha}\right\}$ given in Proposition 1.1. Then, $U\left(f_{\alpha}\right) x \in E_{U, \infty}$ and for $f \in \Phi_{c \delta} U(f) U\left(f_{\alpha}\right) x=U\left(f_{\alpha}\right) U(f) x=0$. Hence, by the above argument, $U\left(f_{\alpha}\right) x \in E_{U, \delta}$. Since $E_{U, \delta}$ is closed, $x=\lim _{\alpha} U\left(f_{\alpha}\right) x \in E_{J, \delta}$.

ExAmPLE 2.1, Let $\Phi$ be an arbitrary basic algebra and let $J_{\lambda}$ be 
the homomorphism of $\Phi$ into $\mathscr{L}(E)$ defined by

$$
J_{\lambda}(f)=f(\lambda) I \text { for all } f \in \Phi(\lambda \in C \text { is fixed }) .
$$

Then, $J_{\lambda}$ is a $\Phi$-spectral representation and

$$
E_{J_{\lambda}, \delta}= \begin{cases}\{0\}, & \text { if } \quad \lambda \notin \delta \\ E, & \text { if } \quad \lambda \in \delta\end{cases}
$$

Hence $\lambda I \in \mathscr{L}(E)$ is $\Phi$-scalar.

EXAMPLE 2.2. Let $\Phi=\mathscr{B}$ and let $U$ be a $\Phi$-spectral representation. If we write $P_{\delta}=U\left(\chi_{\delta}\right)$ for any Borel set $\delta$, where $\chi_{\delta}$ is the characteristic function of $\delta$, then $P_{\delta}$ is a projection on $E$ and $E_{U, \delta}=$ $P_{\delta}(E)$. The family $\left\{P_{\delta} ; \delta\right.$ Borel set $\}$ is a spectral measure in the sense of Dunford or Ionescu Tulcea. (See [2] and [4].)

Proposition 2.3. If $U$ is a $\Phi$-spectral representation and if $\delta$ is a compact set such that $E_{U, \delta} \neq\{0\}$, then

$$
s p\left(U\left(\lambda f_{\delta}\right) / E_{U, \delta}\right) \subseteq \delta,
$$

where $f_{\delta} \in \Phi$ is equal to. 1 in a neighborhood of $\delta$.

Proof. Let $\lambda_{0} \in C \delta$. Choose $f_{\delta} \in \Phi$ such that $f_{\delta}=1$ in a neighborhood of $\delta$ and $f_{\delta}=0$ in a neighborhood $N$ of $\lambda_{0}$. For any $T \in \mathscr{L}(E)$, we write $T_{\delta}=T / E_{\sigma, \delta}$. We know that $U\left(f_{\delta}\right)_{\delta}=I_{\delta}$. Put $S_{\delta}=U\left(\lambda f_{\delta}\right)_{\delta}$ and $f_{\mu}(\lambda)=f_{\delta}(\lambda)(\mu-\lambda)^{-1}$ for $\mu \in N$. Then $f_{\mu} \in \Phi$ and $U\left(f_{\mu}\right)$ is holomorphic in $\mu \in N$, hence so is $U\left(f_{\mu}\right)_{\delta}$. Now, we have

$$
\begin{aligned}
\left(\mu I_{\delta}-S_{\delta}\right) U\left(f_{\mu}\right)_{\delta} & =\left[\left\{\mu U\left(f_{\delta}\right)-U\left(\lambda f_{\delta}\right)\right\} U\left(f_{\mu}\right)\right]_{\delta} \\
& =U\left(\left(\mu f_{\delta}-\lambda f_{\delta}\right)(\mu-\lambda)^{-1} f_{\delta}\right)_{\delta} \\
& =U\left(f_{\delta}^{2}\right)_{\delta}=\left[U\left(f_{\delta}\right)_{\delta}\right]^{2}=I_{\delta} .
\end{aligned}
$$

Therefore, $\lambda_{0} \in \rho\left(S_{\delta}\right)$. Hence, $C \delta \subseteq \rho\left(S_{\delta}\right)$ or $\operatorname{sp}\left(S_{\delta}\right) \subseteq \delta$.

With the help of subspaces $E_{U, \delta}$ and of Proposition 1.3, we are able to extend the notion of $\Phi$-scalar operators to nonregular operators and to non-continuous transformations;

Definition 2.2. A transformation $T$ on $E$ with domain $D_{r}$ is called $\Phi$-scalar if there is a $\Phi$-spectral representation $U$ such that $E_{U, \infty} \subseteq D_{T}$ and $T_{\delta}\left(=T / E_{U, \delta}\right)=U\left(\lambda f_{\delta}\right)$ for any compact set $\delta$, where $f_{\delta} \in \Phi$ is equal to 1 in a neighborhood of $\delta$.

The following proposition is an immediate consequence of the definition: 
Proposition 2.4. If $T$ is $\phi$-scalar, then $T^{n} U(f) \in \mathscr{L}(E)$ for any $n=1,2, \cdots$ and for any $f \in \Phi_{c}$. Furthermore, $T U(f)=U(f) T$ on $E_{U, \infty}$ for any $f \in \Phi$ and $T\left(E_{U, \delta}\right) \subseteq E_{U, \delta}$.

Proposition 2.5. Given any $\Phi$-spectral representation $U$, there is a $\Phi$-scalar closed transformation $T$ to which $U$ is the corresponding representation.

Proof. Using the function $f_{\delta} \in \Phi$ as before, we define $T$ on $E_{U, \delta}$ by $T / E_{U, \delta}=U\left(\lambda f_{\delta}\right) / E_{U, \delta}$ for each compact set $\delta$. Then, $T$ is uniquelly defined on $E_{V, \infty}$ (by Propositions $2.1 \& 2.2$ ). Next, taking a net $\left\{f_{\alpha}\right\}$ given in Proposition 1.1, we define

$$
D_{T}=\left\{x \in E ; \lim _{\alpha} T_{\infty} U\left(f_{\alpha}\right) x \text { exists. }\right\}
$$

and $T x=\lim _{\alpha} T_{\infty} U\left(f_{\alpha}\right) x$ for $x \in D_{T}$. Then we can show that the transformation $T$ with domain $D_{t}$ is closed (in a way similar to the proof of Lemma 2.2 of Bade [1]). This transformation $T$ is obviously $\Phi$-scalar and $U$ is the corresponding $\Phi$-spectral representation.

We remark that the closed transformation constructed above is the minimal closed extension of $T_{\infty}$. Therefore, $\left(T, D_{T}\right)$ is determined independent of the choice of a net $\left\{f_{\alpha}\right\}$.

Proposition 2.6. Suppose $\Phi$ contains constants. Then the statment of Theorem 1.1 remains true even if $\varphi \in \Psi$ is not bounded, i.e., under the same conditions of Theorem $1.1 V(\varphi)$ is $\Phi$-scalar for all $\varphi \in \Psi$. In particular, $U(f)$ is $\Phi$-scalar for all $f \in \Phi$ if $U$ is a $\Phi$-spectral representation and $\Phi$ is either $\mathscr{B}, \mathscr{C}^{\circ}$ or $\mathscr{C}^{\infty}$.

Proof. Let $\delta$ be compact and let $f_{\delta} \in \Phi$ be as before. Then $\left(\lambda f_{\delta}\right) \circ \varphi=\varphi\left(f_{\delta} \circ \varphi\right)$ for any $\varphi \in \Psi$, so that $U\left(\lambda f_{\delta}\right)=V(\varphi) V\left(f_{\delta} \circ \varphi\right)=$ $V(\varphi) U\left(f_{\delta}\right)$. Thus, $U\left(\lambda f_{\delta}\right)_{\delta}=V(\varphi)_{\delta}$.

EXAMPLE 2.3. Unbounded self adjoint operators on Hilbert space, or more generally, unbounded spectral operators of scalar type on Banach space (see [1] and [2]) are $\mathscr{B}$-scalar.

ExAmple 2.4. The operator $T_{A}$ on $C^{\omega}$, given in Example 1 of [5], is $\mathscr{B}$-scalar. If the sequence $A$ is unbounded, then $T_{A}$ is not regular. In fact, $U(f)=T_{f(1)}$, where $f(\Lambda)$ is the sequence $\left\{f\left(\lambda_{1}\right), f\left(\lambda_{2}\right), \cdots\right\}$, is the corresponding $\mathscr{B}$-spectral representation.

EXAmple 2.5. Consider the space $\mathscr{S}=\mathscr{S}\left(R^{n}\right)$ of rapidly decreasing functions on $R^{n}$ given by L. Schwartz [6], i.e., 


$$
\begin{array}{r}
\mathscr{S}=\left\{\varphi \in \mathscr{C}^{\infty}\left(R^{n}\right) ;\left|x^{k} \mathscr{\varphi}^{(p)}(x)\right| \leqq c_{k, p} \text { for all } x \in R^{n}, k \text { and } p\right\} . \\
\left(k=\left(k_{1}, \cdots, k_{n}\right), p=\left(p_{1}, \cdots, p_{n}\right), k_{j}, p_{j}=0,1,2, \cdots\right)
\end{array}
$$

The topology of $\mathscr{S}$ is defined by a countable number or norms $P_{l, m}, l=0,1, \cdots, m=0,1, \cdots$ :

$$
P_{l, m}(\varphi)=\sup \left\{\left|x^{k} \varphi^{(p)}(x)\right| ; x \in R^{n}, 0 \leqq|k| \leqq l, 0 \leqq|p| \leqq m\right\} .
$$

Then $\mathscr{S}$ is a Fréchet space and $\mathscr{L}(\mathscr{S})$ is complete. Let $P=$ $P\left(x_{1}, \cdots, x_{n}\right)$ be a complex valued polynomial in $x_{1}, \cdots, x_{n}$. Corresponding to this polynomial, we consider a linear operator $T_{P}$ on $\mathscr{S}$ defined by $T_{P} \varphi=P \cdot \varphi$ for all $\varphi \in \mathscr{S}$. It can be seen that $T_{P} \in \mathscr{L}(\mathscr{S})$. Also, for $f \in \mathscr{C}_{c}^{\infty}$, we define $U_{P}(f)$ by $U_{P}(f) \varphi=(f \circ P) \varphi$. Again, by a somewhat elaborate computation, we can see that $U_{P}(f) \in \mathscr{L}(\mathscr{S})$ for all $f \in \mathscr{C}_{c}^{\infty}$ and that the mapping $f \rightarrow U_{P}(f)$ is continuous from $\mathscr{C}_{c}^{\infty}$ into $\mathscr{L}(\mathscr{S})$. It is easy to construct a sequence $\left\{f_{n}\right\}$ of functions in $\mathscr{C}_{c}^{\infty}$ such that $\lim _{n \rightarrow \infty} U_{P}\left(f_{n}\right) \varphi=\varphi$ for any $\varphi \in \mathscr{S}$. Thus, $U_{P}$ is a $\mathscr{C}_{c}^{\infty}$ spectral representation. For this $U_{P}, E_{U_{P}, \delta}=\left\{\varphi \in \mathscr{S} ; \operatorname{supp} \varphi \cong P^{-1}(\delta)\right\}$. Then we can see that $T_{P}$ is $\mathscr{C}_{c}^{\infty}$-scalar with the corresponding representation $U_{P}$.

The Fourier transformation $F$ is a topological isomorphism of $\mathscr{S}$ onto itself ([6]. Chap. VII, §6). Let us denote by $\hat{F}$ the induced automorphism on $\mathscr{L}(\mathscr{S}) ; \hat{F} T=F T F^{-1}$ for $T \in \mathscr{L}(\mathscr{S})$. Then, $T$ and $\hat{F} T$ have same spectral properties; in particular, if $T$ is scalar, so is $\hat{F} T$. Since $T_{P}$ is $\mathscr{C}_{c}{ }^{\infty}$-scalar, $\hat{F} T_{P}=P(D)$ is $\mathscr{C}_{c}{ }^{\infty}$-scalar, where $P(D)$ is the differential operator $P\left(D_{1}, \cdots, D_{n}\right), D_{k}=(1 / i)\left(\partial / \partial x_{k}\right)$, on $\mathscr{S}$. Thus, any linear differential operator with constant coefficients is $\mathscr{C}_{c}^{\infty}$-scalar on $\mathscr{S}$. The corresponding $\mathscr{C}_{c}^{\infty}$-spectral representation is, in general, expressed in an integral form. For instance, if $n=1$ and $P(D) \equiv D=$ $(1 / i)(d / d x)$, then

$$
U_{D}(f) \varphi(t)=(1 / 2 \pi) \int_{-\infty}^{\infty} \int_{-\infty}^{\infty} e^{i s(u-t)} f(s, 0) \varphi(u) d s d u
$$

for $f \in \mathscr{C}_{c}^{\infty}$ and $\varphi \in \mathscr{S}(R)$. If $\delta$ is a compact set whose intersection with the real line is a closed interval $[-L, L]$, then

$$
\begin{aligned}
E_{D_{D}, \delta}=\{\varphi \in \mathscr{S}(R) ; \varphi \text { can be extended to an entire } \\
\text { function of exponential type } \leqq L .\}
\end{aligned}
$$

We remark that $s p(P(D))=s p\left(T_{P}\right)=$ image of $P$. Therefore, in particular, $s p(d / d x)=$ the imaginary axis.

3. $\Phi$-spectral transformations. Now that we have the subspaces $E_{U, \delta}$, the following definitions of $\Phi$-spectral operators and $\Phi$-spectral transformations are natural extensions of the spectral operators given by Dunford [2] and Ionescu Tulcea [4]. 
Definition 3.1. Given a basic algebra $\Phi, T \in \mathscr{L}(E)$ is called a $\Phi$ spectral operator if there is a $\Phi$-spectral representation $U$ such that

(i) $T$ commutes with $U$, i.e., $T U(f)=U(f) T$ for all $f \in \Phi$;

(ii) $\operatorname{sp}\left(T / E_{U, \delta}\right) \subseteq \delta$ for any compact set $\delta$ such that $E_{U, \delta} \neq\{0\}$.

Similarly, for a noncontinuous transformation:

DEFINITION 3.2. Given a basic algebra $\Phi$, a transformation $T$ with domain $D_{T} \subseteq E$ is called a $\Phi$-spectral transformation if there is a $\Phi$ spectral representation $U$ such that

(i) $E_{U, \infty} \subseteq D_{R}$;

(ii) $T U(f)=U(f) T$ on $E_{U, \infty}$ for all $f \in \Phi$;

(iii) $T^{n} U(f) \in \mathscr{L}(E)$ for all $n=1,2, \cdots$ and $f \in \Phi_{c}$;

(iv) $s p\left(T / E_{\sigma, \delta}\right) \subseteq \delta$ for any compact set $\delta$ such that $E_{\sigma, \delta} \neq\{0\}$.

Example 3.1. Propositions 2.3, 2.4 and Definition 2.2 imply that any $\Phi$-scalar transformation is $\Phi$-spectral.

EXAMPLE 3.2. Any spectral operator defined by Dunford [2] and by Ionescu Tulcea [4] is $\mathscr{B}$-spectral.

REMARK 1. By the condition (i), we see that $D_{r}$ is dense in $E$.

REMARK 2. If $T$ is closed and if the closed graph theorem holds in $E$, then the condition (iii) follows from (i) and (ii).

REMARK 3. If $E$ is barreled and if we consider the bounded convergence topology in $\mathscr{L}(E)$, then any $\mathscr{B}$-spectral operator $T$ is spectral in the sense of Ionescu Tulcea [4]. Hence, the corresponding representation $U$ is uniquely determined by $T$. On the other hand, Foias [3] showed that $U$ may not be unique if $\Phi=\mathscr{C}_{c}^{\infty}$. Later (Theorem 3.1), we shall show that the spaces $E_{U, \delta}$ are uniquely determined in any case.

Proposition 3.1. If $T$ is $\Phi$-spectral and $U$ is a corresponding $\Phi$-spectral representation, then $\operatorname{supp} U \subseteq \operatorname{sp}(T)$.

Proof. Suppose not, i.e., suppose supp $U \cap \rho(T) \neq \phi$. Let $\mu \in \operatorname{supp} U \cap \rho(T)$ and let $\sigma$ be a neighborhood of $\mu$ contained in $\rho(T)$. Then there is $f \in \Phi_{\sigma}$ such that $U(f) \neq 0$. Then, $E_{U \text {,supp } f} \neq\{0\}$. Since $T$ is $\Phi$-spectral,

$$
s p\left(T / E_{U, \operatorname{supp} f}\right) \subseteq \operatorname{supp} f \cap s p(T)=\phi .
$$

This is impossible. Hence we have the proposition.

COROLlaRY. If $T$ is $\Phi$-spectral and $s p(T)$ is compact, then 
$T \in \mathscr{L}(E)$ (and in fact $T \in \mathscr{L}_{r}(E)$ ).

Proof. If $\operatorname{sp}(T)$ is compact, then supp $U$ is compact by the proposition. Hence $E=E_{U \text {,supp } U} \subseteq D_{T} \subseteq E$, so that $D_{T}=E$.

ExAmple 3.3. Let $J_{\lambda}(\lambda \in C$ : fixed) be the $\Phi$-spectral representation given in Example 2.1. Then $T$ is $\Phi$-spectral with respect to $J_{\lambda}$ if and only if $T \in \mathscr{L}(E)$ and $\operatorname{sp}(T)=\{\lambda\}$.

Proof. Let $T \in \mathscr{L}(E)$ and $s p(T)=\{\lambda\}$. Obviously, $T$ commutes with $J_{\lambda}$. If $E_{J_{\lambda}, \delta} \neq\{0\}$, then $\lambda \in \delta$ and $E_{J_{\lambda}, \delta}=E$. Then, $s p\left(T / E_{J_{\lambda}, \delta}\right)=s p(T)=$ $\{\lambda\} \subseteq \delta$. Therefore, $T$ is $\Phi$-spectral with respect to $J_{\lambda}$. Conversely if $T$ is $\Phi$-spectral with respect to $J_{\lambda}$, then $s p(T)=s p\left(T / E_{J_{\lambda},\{\lambda\}}\right) \subseteq\{\lambda\}$, since $E_{J_{\lambda},\{\lambda\}}=E . \quad s p(T)$ being nonempty, $s p(T)=\{\lambda\} . \quad T \in \mathscr{L}(E)$ by the above corollary. Thus, in this case, $T=\lambda I+Q$, where $Q$ is a quasinilpotent operator.

Proposition 3.2. Let $T$ be $\Phi$-spectral and let $U$ be a corresponding $\Phi$-spectral representation. If $(\lambda I-T) x=0$ for some $\lambda \in C$ and $x \in D_{T}$, then $x \in E_{U,\{\lambda\}}$ and

$U(f) x=0$ for any $f \in \Phi$ such that $\lambda \notin \operatorname{supp} f$;

$U(f) x=0$ for any $f \in \Phi$ such that $f \equiv 1$ in a neighborhood of $\lambda$.

Proof. Let $\delta=\operatorname{supp} f$ be compact and let $\lambda \notin \delta . \quad$ Since $s p(T) \leqq \delta$, there is $R_{\lambda, \delta}$ such that

$$
(\lambda I-T)_{\delta} R_{\lambda, \delta}=R_{\lambda, \delta}(\lambda I-T)_{\delta}=I_{\delta} .
$$

Since $U(f) x \in E_{U, \delta}$, we have

$$
U(f) x=R_{\lambda, \delta}(\lambda I-T) U(f) x=R_{\lambda, \delta} U(f)(\lambda I-T) x=0 .
$$

Since $\Phi_{c}$ is dense in $\Phi, U(f) x=0$ even if $\operatorname{supp} f$ is not compact. Hence, we conclude that $x \in E_{U,\{\lambda]}$ by Proposition 2.2. The last statement of the proposition follows from Proposition 2.1.

Proposition 3.3. If $T$ is $\Phi$-spectral, then it has the single valued extension property, i.e., if we have

$$
(\lambda I-T) x_{\lambda}=(\lambda I-T) y_{\lambda}=x,
$$

where $x_{\lambda}$ and $y_{\lambda}$ are holomorohic in open sets $\sigma_{1}$ and $\sigma_{2}$ respectively, then $x_{\lambda}=y_{\lambda}$ on $\sigma_{1} \cap \sigma_{2}$.

Proof. We can prove this in a way similar to the proof of Theorem 1 in Foias [3], using the previous proposition, 
We are now able to define the spectrum of an element $x \in E$ with respect to a $\Phi$-spectral transformation $T$, as was done in Dunford [2] and Foias [3] for special cases:

Definition 3.3. Let $T$ be $\Phi$-spectral and let $x \in E$. The set of all $\lambda_{0} \in \hat{C}$ such that there is a holomorphic function $x_{\lambda}\left(\in D_{T}\right)$ defined in a neighborhood $N$ of $\lambda_{0}$ satisfying the equation $(\lambda I-T) x_{\lambda}=x$ for $\lambda \in N-\{\infty\}$, will be denoted by $\rho_{T}(x)$. The set $s p_{T}(x)=\widehat{C}-\rho_{T}(x)$ is called the spectrum of $x$ with respect to $T$.

By the previous proposition, $x_{\lambda}$ is uniquely determined on $\rho_{T}(x)$ and $x_{\lambda}=(\lambda I-T)^{-1} x$ if $\lambda \in \rho(T)-\{\infty\}$. Therefore, $s p_{T}(x) \leqq s p(T)$. Obviously, $s p_{T}(x)$ is closed in $\hat{C}$.

Theorem 3.1. If $T$ is $\Phi$-spectral and $U$ is a corresponding $\Phi$ spectral representation, then $E_{U, \delta}=\left\{x ; s p_{T}(x) \subseteq \delta\right\}$ for any compact set $\delta$. Therofore, $E_{T, \delta}=E_{U, \delta}$ is uniquely determined by $T$.

The proof of this theorem is again similar to those of Proposition 3 and Theorem 2 of Foias [3]. If we use our Proposition 2.2, then the proof will become simpler.

4. Decomposition theorem for $\Phi$-spectral transformations. In what follows, we always assume that $T$ is a $\Phi$-spectral transformation, $U$ is a corresponding $\Phi$-spectral representation and $E_{\delta}=E_{T, \delta}=E_{U, \delta}$ is the subspace of $E$ for each compact set $\delta$, given in the previous sections.

LEMma 1. Let $\delta$ be a compact set such that $E_{\delta} \neq\{0\}$. Given $\lambda \in C \delta$, let $\rho_{\lambda}=\sup _{\mu \in \delta}|\mu-\lambda|$. Then, for any $x \in E_{\delta}, r>\rho_{\lambda}$, the set $\left\{(\lambda I-T)^{n} x / r^{n} ; n=1,2, \cdots\right\}$ is bounded in $E$.

Proof. Let $\lambda_{0} \in C \delta$ be fixed. Since $x \in E_{\delta}, s p_{T}(x) \subseteq \delta$ by Theorem 3.1. Hence, there is a holomorphic function $x_{\lambda}$ defined outside of $\delta$, satisfying $(\lambda I-T) x_{\lambda}=x$. In particular, $x_{\lambda}$ is holomorphic for $\left|\lambda-\lambda_{0}\right|>\rho_{\lambda_{0}}$, so that

$$
x_{\lambda}=\sum_{n=0}^{\infty} \frac{\left(\lambda_{0} I-T\right)^{n} x}{\left(\lambda-\lambda_{0}\right)^{n+1}}
$$

for $\left|\lambda-\lambda_{0}\right|>\rho_{\lambda_{0}}$ and the series converges uniformly for $\left|\lambda-\lambda_{0}\right| \geqq r$, $r>\rho_{\lambda_{0}}$ (see, for example, Dunford [2]). Therefore, we have the proposition.

CoROLlary. Let supp $f$ be compact, $\lambda \notin \operatorname{supp} f$ and let $\rho_{f, \lambda}=$ $\sup _{\mu \in \operatorname{supp} f}|\mu-\lambda|$. Then, for any $x \in E$ and $r>\rho_{f, \lambda}$, the set 
$\left\{(\lambda I-T)^{n} U(f) x / r^{n} ; n=1,2, \cdots\right\}$ is bounded in $E$.

Proof. If $U(f) x=0$, then it is trivial. If $U(f) x \neq 0$, then $E_{\text {supp } f} \neq$ $\{0\}$, so that we can apply the lemma for $U(f) x$ and $\delta=\operatorname{supp} f$.

Given a compact set $\delta$, let $f_{\delta} \in \Phi_{c}$ be equal to 1 in a neighborhood $\sigma$ of $\delta$.

Lemma 2. Let $f \in \Phi$ and supp $f \subset \sigma$. Then, for any $g \in \Phi$, the set $\left\{\left[T-U\left(\lambda f_{\delta}\right)\right]^{n} U(f) U(g) / r^{n} ; n=1,2, \cdots\right\}$ is bounded in $\mathscr{L}(E)$ for every $r>2 d_{g}$, where $d_{g}$ is the diameter of supp $g$.

Proof. First, let us remark that

$$
U\left(f_{\delta}\right)^{k} U(f)=U(f)
$$

and

$$
U\left(\lambda f_{\delta}\right)^{k} U(f)=U\left(\lambda^{k} f\right)
$$

Now, we can write

$$
\begin{aligned}
{\left[T-U\left(\lambda f_{\delta}\right)\right]^{n} U(f) U(g) } \\
\quad=\left\{\left[\lambda_{0} I-U\left(\lambda f_{\delta}\right)\right]-\left(\lambda_{0} I-T\right)\right\}^{n} U(f) U(g) \\
\quad=\sum_{k=0}^{n}(-1)^{k}\left(\begin{array}{l}
n \\
k
\end{array}\right)\left[\lambda_{0} I-U\left(\lambda f_{\delta}\right)\right]^{k}\left(\lambda_{0} I-T\right)^{n-k} U(f) U(g) \\
\quad=\sum_{k=0}^{n}(-1)^{k}\left(\begin{array}{l}
n \\
k
\end{array}\right) U\left[\left(\lambda_{0}-\lambda\right)^{k} f\right]\left(\lambda_{0} I-T\right)^{n-k} U(g) .
\end{aligned}
$$

Let $r_{1}=r / 2$ and let $f_{1} \in \Phi$ be such that $f_{1}=1$ on a neighborhood of supp $g$ and $d_{f_{1}}<r_{1}{ }^{1}$ Then, since $U(g) U\left(f_{1}\right)=U(g)$,

$$
\begin{aligned}
{[T-} & \left.U\left(\lambda f_{\delta}\right)\right]^{n} U(f) U(g) / r^{n} \\
& =\left(1 / 2^{n}\right) \sum_{k=0}^{n}(-1)^{k}\left(\begin{array}{l}
n \\
k
\end{array}\right)\left\{U\left[\left(\lambda_{0}-\lambda\right)^{k} f \cdot f_{1}\right] / r_{1}^{k}\right\}\left\{\left(\lambda_{0} I-T\right)^{n-k} U(g) / r_{1}{ }^{n-k}\right\} .
\end{aligned}
$$

If $\lambda \in \operatorname{supp} f \cdot f_{1} \subseteq \operatorname{supp} f_{1}$, then $\left|\lambda_{0}-\lambda\right|<\rho_{f_{1}, \lambda_{0}}<r_{1}$. Hence, the set $\left\{\left(\lambda_{0}-\lambda\right)^{k} f \cdot f_{1} / r_{1}^{k} ; k=0,1, \cdots\right\}$ is bounded in $\Phi$ by (iii) of Definition 1.1. Hence, $B_{1}=\left\{U\left[\left(\lambda_{0}-\lambda\right)^{k} f \cdot f_{1}\right] / r_{1}^{k} ; k=0,1, \cdots\right\}$ is bounded in $\mathscr{L}(E)$.

On the other hand, since $\rho_{g, \lambda_{0}}<\rho_{f_{1}, \lambda_{0}}<r_{1}$, it follows from the above corollary that the set $\left\{\left(\lambda_{0} I-T\right)^{k} U(g) x / r_{1}^{k} ; k=0,1, \cdots\right\}$ is bounded for all $x \in E$. Then, $B_{2}=\left\{\left(\lambda_{0} I-T\right)^{k} U(g) / r_{1}^{k} ; k=0,1, \cdots\right\}$ is bounded in $\mathscr{L}(E)$. The set $\left\{\left[T-U\left(\lambda f_{\delta}\right)\right]^{n} U(f) U(g) / r^{n} ; n=1,2, \cdots\right\}$ is contained in the convex hull of $B_{1} B_{2}$, which is again bounded. Hence the set is bounded and the lemma is proved.

We are now ready to prove the following proposition, to which the main theorem of decomposition is a corollary:

${ }_{1}^{1}$ We choose $\lambda_{0} \in C \operatorname{supp} f_{1}$ such that $\rho_{f_{1}, \lambda_{0}}<r_{1}$. 
Proposition 4.1. Given a compact set $\delta$ and $f_{\delta} \in \Phi$, if supp $f \subseteq \sigma$, $f \in \Phi$, then the set

$$
\left\{\left[T-U\left(\lambda f_{\delta}\right)\right]^{n} U(f) / \varepsilon^{n} ; n=1,2, \cdots\right\}
$$

is bounded in $\mathscr{L}(E)$ for any $\varepsilon>0$.

Proof. Given $\varepsilon>0$, we can construct (by (i) of Definition 1.1) a finite $\varepsilon / 2$-decomposition of unity on $\delta$, i.e., a finite set of functions $\left\{h_{k}\right\}_{k=1,2}, \cdots, l, h_{k} \in \Phi$, such that the diameter of each supp $h_{k}$ is less than $\varepsilon / 2, \sum h_{k} \equiv 1$ in a neighborhood of $\delta$. Then, we have

$$
\left[T-U\left(\lambda f_{\delta}\right)\right]^{n} U(f) / \varepsilon^{n}=\sum_{k=1}^{l}\left\{\left[T-U\left(\lambda f_{\delta}\right)\right]^{n} U(f) U\left(h_{k}\right) / \varepsilon^{n}\right\} .
$$

By Lemma 2, the set $\left\{\left[T-U\left(\lambda f_{\delta}\right)\right]^{n} U(f) U\left(h_{k}\right) / \varepsilon^{n} ; n=1,2, \cdots\right\}$ is bounded for each $k$, since $\varepsilon>2 d_{h_{k}}$. Hence, we obtain the proposition.

THEOREM 4.1. Any $\Phi$-spectral transformation $T$ can be expressed $T=S+Q$ on $E_{T, \infty}$, where $S$ is a $\Phi$-scalar transformation and $Q$ is a transformation defined on $E_{r^{\prime}, \infty}$ such that $\lim _{n \rightarrow \infty}\left|\left\langle Q^{n} x, x^{\prime}\right\rangle\right|^{1 / n}=0$ for all $x \in E_{r, \infty}$ and $x^{\prime} \in E^{\prime}$. If $T$ is closed, then $S$ and $Q$ can be taken as closed transformations with domains containing $E_{T, \infty}$.

Proof. Let $U$ be a corresponding $\Phi$-spectral representation to $T$ and let $S$ be the closed $\Phi$-scalar transformation constructed in Proposition 2.5. Let $Q=T-S$ on $D_{Q}=D_{T} \cap D_{S}$. Then if $T$ is closed, so is $Q$. For any $x \in E_{r, \infty}$, there is a compact set $\delta$ such that $x \in E_{r, \delta}$. Then, $S x=U\left(\lambda f_{\delta}\right) x$. Hence, taking $f \in \Phi_{c}$ such that supp $f \subset \sigma$ and expressing $x=U(f) y$, we have $Q^{n} x=\left[T-U\left(\lambda f_{\delta}\right)\right]^{n} U(f) y$. Therefore, it follows from Proposition 4.1 that $\lim _{n \rightarrow \infty}\left|\left\langle Q^{n} x, x^{\prime}\right\rangle\right|^{1 / n}=0$ for all $x^{\prime} \in E^{\prime}$.

REMARK 1. The example of Bade [1] shows that

$$
\lim _{n \rightarrow \infty}\left|\left\langle Q^{n} x, x^{\prime}\right\rangle\right|^{1 / n}=0
$$

might not be satisfied for all $x \in D_{Q}$.

REMARK 2. If $\Phi=\mathscr{S}$, then $S$, hence $Q$, are uniquely determined by $T$, provided that $E$ is barreled. But it is not true if $\Phi$ is arbitrary. (See Remark 3 in the previous section.)

Corollary. If $T$ is $\Phi$-spectral and $s p(T)$ is compact, then $T=$ $S+Q$ where $S$ is a $\Phi$-scalar and $Q$ is a quasi-nilpotent operator.

A converse theorem can be given in the following form: 
THEOREM 4.2. Let $S$ be a $\Phi$-scalar transformation with a corresponding $\Phi$-specral representation $U$. Let $Q$ be a transformation such that $E_{S, \infty} \subseteq D_{Q}, Q U(f)=U(f) Q$ on $E_{S, \infty}, Q^{n} U(f) \in \mathscr{F}(E)$ for all $f \in \Phi_{c}$ and $\lim _{n \rightarrow \infty}\left|\left\langle Q^{n} x, x^{\prime}\right\rangle\right|^{1 / n}=0$ for any $x \in E_{S, \infty}, x^{\prime} \in E^{\prime}$. Then $T=S+Q$ defined on $D_{T}=D_{S} \cap D_{Q}$ is a $\Phi$-spectral transformation.

Proof. It is enough to show that $s p\left(T_{\delta}\right) \subseteq \delta$ for any compact set $\delta$. For any $\lambda_{0} \in C \delta$, let $f \in \Phi_{c}$ be equal to 1 in a neighborhood of $\delta$, equal to 0 in a neighborhood $N$ of $\lambda_{0}$. Since $U(f) x \in E_{s, \infty}$ for any $x \in E$, $\lim _{n \rightarrow \infty}\left|\left\langle Q^{n} U(f) x, x^{\prime}\right\rangle\right|^{1 / n}=0$ for all $x \in E, x^{\prime} \in E^{\prime}$. Then, the series

$$
R_{\mu}=\sum_{k=0}^{\infty} Q^{k} U\left[(\mu-\lambda)^{-k-1} f\right]
$$

converges uniformly for $\left|\mu-\lambda_{0}\right| \leqq \varepsilon_{0}$ for some $\varepsilon_{0}>0$. (Note that $(\mu-\lambda)^{-k-1} f(\lambda) \in \Phi$ for all $k$ and each term of the series belongs to $\mathscr{L}(E)$.) Since $\mathscr{L}(E)$ is quasi-complete, $R_{\mu} \in \mathscr{L}(E)$ and is holomorphic in $\mu:\left|\mu-\lambda_{0}\right| \leqq \varepsilon_{0}$. It is easy to see that $R_{\mu}(\mu I-S-Q) U(f) x=$ $(\mu I-S-Q) R_{\mu} U(f) x=x$ for all $x \in E_{S, \delta}$. Therefore, $R_{\mu, \delta}$ is the inverse of $\mu I_{\delta}-T_{\delta}$ on $E_{S, \delta}$ and $R_{\mu, \delta}$ is holomorphic in a neighborhood of $\lambda_{0}$. Hence, $\lambda_{0} \in \rho\left(T_{\delta}\right)$ or $C \delta \subseteq \rho\left(T_{\delta}\right)$, i.e., $s p\left(T_{\delta}\right) \subseteq \delta$.

\section{REFERENCES}

1. W. G. Bade, Unbounded spectral operators, Pacific J. Math., 4 (1954), 373-392.

2. N. Dunford, A survey of the theory of spectral operators, Bull. Am. Math. Soc., 64 (1958), 217-274.

3. M. C. Foias, Une application des distributions vectorielles à la théorie spectrale, Bull. Sc. Math. $2^{e}$ série, 84 (1960), 147-158.

4. C. Ionescu Tulcea, Spectral operators on locally convex spaces, Bull. Am. Math. Soc., 67 (1961), 125-128.

5. Fumiyuki Maeda, Remarks on spectra on operators on a locally convex space, Proc. Nat. Acad. Sci., 47 (1961), 1052-1055.

6. L. Schwartz, Théorie des distributions, I \& II, Paris, 1951.

7. L. Waelbroeck, Le calcul symbolique dans les algèbres commutative, J. de Math., $9^{e}$ série, 33 (1954), 147-186. 


\section{PACIFIC JOURNAL OF MATHEMATICS}

\section{EDITORS}

\section{RalPh S. Phillips}

Stanford University

Stanford, California

M. G. Arsove

University of Washington

Seattle 5, Washington
J. Dugundji

University of Southern California Los Angeles 7, California

Lowell J. Paige

University of California

Los Angeles 24, California

\section{ASSOCIATE EDITORS}
E. F. BECKENBACH
D. DERRY
H. L. ROYDEN
E. G. STRAUS
T. M. CHERRY
M. OHTSUKA
E. SPANIER
F. WOLF

\section{SUPPORTING INSTITUTIONS}

\author{
UNIVERSITY OF BRITISH COLUMBIA \\ CALIFORNIA INSTITUTE OF TECHNOLOGY \\ UNIVERSITY OF CALIFORNIA \\ MONTANA STATE UNIVERSITY \\ UNIVERSITY OF NEVADA \\ NEW MEXICO STATE UNIVERSITY \\ OREGON STATE UNIVERSITY \\ UNIVERSITY OF OREGON \\ OSAKA UNIVERSITY \\ UNIVERSITY OF SOUTHERN CALIFORNIA
}

STANFORD UNIVERSITY

UNIVERSITY OF TOKYO

UNIVERSITY OF UTAH

WASHINGTON STATE UNIVERSITY

UNIVERSITY OF WASHINGTON

AMERICAN MATHEMATICAL SOCIETY

CALIFORNIA RESEARCH CORPORATION SPACE TECHNOLOGY LABORATORIES

NAVAL ORDNANCE TEST STATION

Printed in Japan by International Academic Printing Co., Ltd., Tokyo Japan 


\section{Pacific Journal of Mathematics}

\section{Vol. 13, No. 1 \\ March, 1963}

Frantz Woodrow Ashley, Jr., A cone of super-(L) functions............. 1

Earl Robert Berkson, Some metrics on the subspaces of a Banach space....

Felix Earl Browder and Walter Strauss, Scattering for non-linear wave

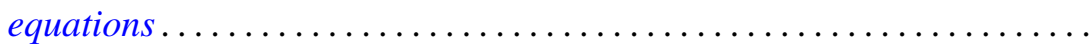

Edmond Darrell Cashwell and C. J. Everett, Formal power series ..........

Frank Sydney Cater, Continuous linear functionals on certain topological

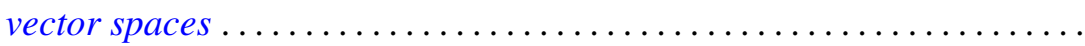

John Douglas Dixon, General group extensions ....................

Robert Pertsch Gilbert, On harmonic functions of four variables with

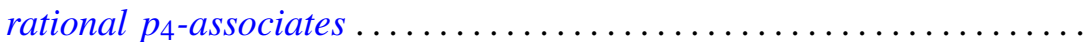

Irving Leonard Glicksberg, On convex hulls of translates ..............

Simon Hellerstein, On a class of meromorphic functions with deficient zeros

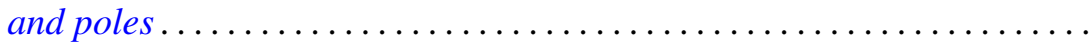

Donald William Kahn, Secondary cohomology operations which extend the

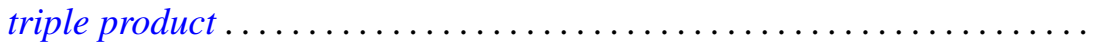

G. K. Leaf, A spectral theory for a class of linear operators .............

R. Sherman Lehman, Algebraic properties of the composition of solutions of partial differential equations ........................... 157

Joseph Lehner, On the generation of discontinuous groups ............. 169

S. P. Lloyd, On certain projections in spaces of continuous functions ...... 171 Fumi-Yuki Maeda, Generalized spectral operators on locally convex spaces ..................................

Donald Vern Meyer, $E^{3}$ modulo a 3-cell

William H. Mills, An application of linear programming to permutation groups.

Richard Scott Pierce, Centers of purity in abelian groups

Christian Pommerenke, On meromorphic starlike functions ...

Zalman Rubinstein, Analytic methods in the study of zeros of

polynomials...

B. N. Sahney, On the Nörlund summability of Fourier series

Tôru Saitô, Regular elements in an ordered semigroup . .

Lee Meyers Sonneborn, Level sets on spheres...........

Charles Andrew Swanson, Asymptotic estimates for limit point

problems .

Lucien Waelbroeck, On the analytic spectrum of Arens . .

Alvin (Murray) White, Singularities of a harmonic function of three

variables given by its series development .............

Kōichi Yamamoto, Decomposition fields of difference sets ...

Chung-Tao Yang, On the action of $\mathrm{SO}(3)$ on a cohomology manifold... 